\title{
Erratum: Comparative study of the interfaces of graphene and hexagonal boron nitride with silver [Phys. Rev. B 94, 155431 (2016)]
}

\author{
Manuela Garnica, Martin Schwarz, Jacob Ducke, Yuanqin He, Felix Bischoff, \\ Johannes V. Barth, Willi Auwärter, and Daniele Stradi
}

(Received 7 February 2019; published 25 February 2019)

DOI: 10.1103/PhysRevB.99.089901

We have checked our scanning tunneling spectroscopy data of the $h-\mathrm{BN} / \mathrm{Ag} / \mathrm{Cu}(111)$ system and realized that we assigned an incorrect number of $\mathrm{Ag}$ intercalated monolayers (MLs). In the original paper, we concluded that the copper surface was covered by $3 \mathrm{ML}$ of silver [Fig. 1(b), Fig. S1, Table I). It has been previously demonstrated (Ref. [47]) that the Ag film thickness on $\mathrm{Cu}(111)$ can be determined by the position of the surface state onset. In our case, the value of the band minimum on $\mathrm{Ag} / \mathrm{Cu}(111)$ is $-235 \mathrm{mV}$ [Fig. 2(d)], which corresponds to only $1 \mathrm{ML}$. Accordingly, assuming a constant Ag thickness below $h$-BN, the intercalated Ag film is $1 \mathrm{ML}$ throughout the paper (even though we also performed experiments on $3 \mathrm{ML}$ thick Ag films). Other than that, the scientific ideas and conclusions of the original paper are not affected by this Erratum.

[47] M. Wessendorf, C. Wiemann, M. Bauer, M. Aeschlimann, M. A. Schneider, H. Brune and K. Kern, Appl. Phys. A: Mater. Sci. Process. 78, 183 (2004). 